\title{
Líneas de acción para prevenir y controlar la delincuencia juvenil en comunidades vulnerables de Barranquilla-Colombia- y su área metropolitana*
}

\section{Actions to prevent and control the juvenile delinquency in vulnerable communities in Barranquilla and its metropolitan area}

\author{
DAVID LIBREROS ORTEGA \\ Técnico Profesional en Servicio de Policía. Jefe del Área de Investigación. Escuela de Policía Antonio Nariño (ESANA). \\ david.libreros@correo.policia.gov.co \\ Avenida Circunvalar No. 45 - 30 \\ Barranquilla-Colombia
}

ZOILO ASPRILLA LARA

Técnico Profesional en Servicio de Policía. Coordinador del Programa Jóvenes a lo Bien. Policía Nacional, Metropolitana de Barranquilla (MEBAR)

zoilo.asprilla@correo.policia.gov.co

MARITZA TURIZO ARZUZA

Licenciada en Ciencias de la Educación; Magister en Docencia e Investigación Universitaria; Diploma de Estudios Avanzados por Doctorado en Lengua Española y Lingüística General; Doctora (C) Ciencias de la Educación. Asesora metodológica en el Área de Investigación, Escuela de Policía Antonio Nariño (ESANA).

matarzu@gmail.com

maturizo@yahoo.com

Para citar este artículo

Libreros Ortega, D; Asprilla Lara, Z \& Turizo Arzuza, M (2015). Líneas de acción para prevenir y controlar la delincuencia juvenil en comunidades vulnerables de Barranquilla-Colombia- y su área metropolitana Justicia Juris, 11(1), 40-51.

Recibido: Diciembre 13 de 2014

Aceptado: Febrero 26 de 2015

RESUMEN

Este artículo está basado en una investigación cuyo objetivo general ha sido caracterizar el fenómeno de la delincuencia juvenil en la ciudad de Barranquilla y su área metropolitana, a partir de la identificación del perfil de los jóvenes infractores, factores de riesgo, necesidades de programas, planes y estrategias de atención, prevención y control de este flagelo. Para ello, se acudió a un enfoque crítico social y un diseño

\footnotetext{
* Artículo científico resultado del proyecto de investigación institucional "Caracterización y prevención de la delincuencia juvenil en Barranquilla y su área Metropolitana", ejecutado por el Grupo de Investigación y Semilleros ESANA - DINAE de la Escuela de Policía Antonio Nariño (ESANA), en colaboración con el Programa Jóvenes a lo Bien de la Policía Metropolitana de Barranquilla (MEBAR). Se agradece el apoyo a: Dirección y Subdirección de la ESANA, Vicerrectoría de investigaciónDirección Nacional de Escuelas (VICIN-DINAE), Alcaldía de Barranquilla, Gobernación del Atlántico, ACOPI, y SENA. La investigación se desarrolló durante el período Febrero - Noviembre 2013 y su implementación se extendió al año 2014.
} 
de Investigación Acción Participación delimitado en cuatro fases: descriptiva, propositiva, intervención y evaluación. Aunque el punto de partida fue una encuesta diagnóstica a 250 informantes en los cinco sectores de la ciudad y municipios del área metropolitana; el eje metodológico lo constituyó el trabajo colaborativo intersectorial que se desarrolló con representantes de las autoridades locales y de la comunidad afectada. De tal modo, además de delinear el perfil del delincuente juvenil y obtener de la comunidad una valoración de las políticas y programas ejecutados por los estamentos públicos y privados; se delimitaron las líneas de acción que se considerarían fundamentales para contrarrestar el fenómeno. Finalmente, dado el análisis de potenciales logros y dificultades, a partir de la experiencia que cada uno de los miembros del colectivo tenía en el tema; la propuesta se focalizó en tres aspectos centrales: Familia, Comunidad y Escuela; y su implementación cubriría en primer lugar, las zonas más vulnerables a la delincuencia juvenil en Barranquilla y su área metropolitana.

Palabras Clave: líneas de acción, prevención, control, delincuencia juvenil, comunidad vulnerable, trabajo colaborativo intersectorial, caracterización, violencia.

\begin{abstract}
This paper is based on a research whose general aim is to characterize the phenomenon of juvenile delinquency in the city of Barranquilla and its metropolitan area, from the identification of the profile of young offenders, risk factors, needs programs, plans and strategies of care, prevention and control of this flagellum. To do this, it was convenient to assume a social critical approach and a Participation Action Research designed and delimited in four phases: descriptive, purposeful, intervention and evaluation. Although the starting point was a diagnostic survey of 250 respondents in the five sectors of the city of Barranquilla and its municipalities of the metropolitan area; methodological axis was conducted by representatives of local authorities by those sectors along with the affected community. Thus, in addition to delineate the profile of juvenile community and get an assessment of policies and programs implemented by public and private entities; actions that are considered essential to counter the phenomenon were defined. Finally, since the analysis of potential achievements and difficulties, from the experience that each of the members of the group had on the subject; the proposal focused on three core areas: Family, Community and School; and its implementation would cover first, most vulnerable areas to juvenile delinquency in Barranquilla and its metropolitan area.
\end{abstract}

Keywords: lines of action, prevention, control, juvenile delinquency, vulnerable community, intersectoral collaborative work, characterization, violence.

\section{Introducción}

La inseguridad en las calles de Barranquilla-Colombia- y su área metropolitana ha ido en ascenso y es así como hoy se registra un alto índice de delitos que potencia y diversifica una violencia que mantiene en vilo a sus habitantes. De hecho, cada vez son más habituales las contravenciones que implican la presencia injustificada de acciones extremadamente violentas.

De tal manera, las faltas contra el patrimonio, constituyen la mayor cantidad de infracciones penales que se produce en la capital del Departamento del Atlántico especialmente, lo cual es un síntoma inequívoco de que el fenómeno delincuencial ha aumentado cuantitativa y cualitativamente, especialmente en lo que se refiere a robos y lesiones graves. Es así como suelen presentarse atracos a bancos, a residencias y a sujetos en las calles; hechos que a menudo se acompañan de acciones que ponen en riesgo la vida e integridad de los ciudadanos.

Otra característica de la delincuencia que hoy afronta esta urbe del Caribe colombiano, es la existencia de grupos de maleantes profesionales constituidos en torno a movimientos ilegales que les representan beneficios económicos. Estos operan bajo la etiqueta de bandas delincuenciales que bien pueden no tener una tipología definida de delito, o especializarse en asesinatos por encargo, secuestro, extorsión y otros sectores de actividad punible. Junto a estos grupos fuertemente estructurados y jerarquizados, existen otros que ejercen su actividad en algún sector delincuencial: traficantes de drogas, equipos especializados en determinados tipos de robos, estafa, tráfico de moneda falsa, trata de personas y tráfico de armas, entre un sinnúmero de actos delictivos. Entre ellos, no parece existir relaciones orgánicas sino episódicas, con 
ocasión de un asunto determinado, de los cuales pueden resultar relaciones conflictivas que desembocan en ajustes de cuentas a manos de sicarios que se desplazan desde otras ciudades hasta Barranquilla en busca de su víctima.

Se requiere por tanto, que la Policía Nacional ejerza a cabalidad su misión, y en tal medida, demuestre que su papel en la lucha contra la delincuencia es preponderante. Más aún que sus miembros hagan visible su labor para intervenir en la caracterización y prevención del flagelo, sobre todo en ciudades como Barranquilla, donde se ha logrado detectar que cada día más, los jóvenes se muestran propensos a delinquir, incluso antes de haber llegado a la mayoría de edad.

En razón de lo expresado anteriormente, en febrero de 2013, la Escuela de Policía Antonio Nariño (ESANA), en colaboración con el Programa Jóvenes a lo Bien de la Policía Metropolitana de Barranquilla (MEBAR) y contando con el apoyo de las autoridades locales y departamentales, emprendió una investigación cuyo problema quedó sintetizado en el siguiente interrogante central: ¿De qué manera los componentes y aspectos que caracterizan la delincuencia juvenil en Barranquilla y su área metropolitana son referentes para el desarrollo de un proceso participativo dirigido a la prevención del fenómeno en comunidades vulnerables?

En coherencia con el problema formulado y el enfoque epistemológico- metodológico adoptado; se cumplió con el objetivo general trazado, consistente en caracterizar el fenómeno de la delincuencia juvenil en la ciudad de Barranquilla y su área metropolitana, a partir de la identificación del perfil de los jóvenes infractores, factores de riesgo asociados y necesidades en materia de programas, planes y estrategias de atención, prevención y control de este flagelo, tomando como base el trabajo colaborativo entre la policía nacional, los representantes de los sectores públicos y privados, y los líderes de grupos o comunidades vulnerables.

En referencia al contexto de Barranquilla y su área metropolitana, para alcanzar la meta de investigación, el proyecto apuntó a tres objetivos específicos:

- Identificar el perfil de los jóvenes infractores y su asociación a factores de riesgo para conductas delictivas.

- Reseñar las características, alcances y resultados de las políticas y acciones imple- mentadas por las comunidades y estamentos públicos y privados para contrarrestar la delincuencia juvenil.

- Delimitar mediante un trabajo colaborativo -intersectorial y comunitario- liderado por la policía nacional, las líneas de acción para el diseño e implementación de programas, planes y estrategias de atención, prevención y control de la delincuencia juvenil en contextos de alta vulnerabilidad.

Lo anterior, se logró fundamentar gracias a la revisión de la literatura que se efectuó, ya que fue de vital importancia conocer en primer lugar el término delincuencia y su constitución a nivel general, debido a que radica en un grave problema social de difícil prevención pero de aún más difícil solución. El término delincuencia es un concepto de carácter legal, social, psicológico y moral. En este sentido, Monroy (1998) afirma que "el crecimiento de la delincuencia juvenil, como una violenta explosión del drama que vive el mundo actual, figura entre los problemas que más preocupan a los gobiernos de hoy" (p.123). Es por ello, que se considera la delincuencia juvenil como un fenómeno que de forma alarmante ha aumentado en los últimos tiempos, pasando a ser un problema que cada vez genera mayor preocupación social, tanto por su incremento cuantitativo como por su progresiva peligrosidad cualitativa.

En este sentido, resulta pertinente apuntar al perfil de ese delincuente juvenil, el cual se ve específicamente como

$$
\begin{aligned}
& \text {...una figura cultural, porque su definición } \\
& \text { y tratamiento legal responden a distintos } \\
& \text { factores en distintas naciones, reflejando una } \\
& \text { mezcla de conceptos psicológicos y lega- } \\
& \text { les. Técnicamente, el delincuente juvenil es } \\
& \text { aquella persona que no posee la mayoría de } \\
& \text { edad penal y que comete un hecho que está } \\
& \text { castigado por las leyes. (Garrido, 1986, p.11) }
\end{aligned}
$$

Cabe anotar que esta problemática, que se da en la cotidianidad, tiene un espacio en el seno de la Asamblea General de la Organización de Naciones Unidas (ONU). En esta, se ha discutido asiduamente el tema de la delincuencia de menores, hasta el punto, que se han adoptado dos instrumentos de gran trascendencia para el procesamiento judicial y, cuando a ello haya lugar, la privación de la libertad de los menores infractores de la ley penal. Son estas las Reglas Mínimas de las Naciones Unidas para la administración de la justicia de menores, conocidas como "Reglas de Beijing" (aprobadas 
mediante Resolución 40/33 del 28 de noviembre de 1985), y las Reglas de las Naciones Unidas para la protección de los menores privados de libertad -aprobadas mediante Resolución 45/113 del 14 de diciembre de 1990- (Nueva Ley para la Infancia y la Adolescencia en Colombia, 2004).

De acuerdo con lo anteriormente esbozado, se hizo necesario, revisar los antecedentes científicos, relacionados con el tema de la delincuencia en diferentes contextos. En primera instancia, se encontró la investigación realizada por Páez Morales (2013), a través de la cual se pretendió demostrar cómo la delincuencia juvenil específicamente en los colegios oficiales de la ciudad de Bogotá D.C, afecta la convivencia y seguridad ciudadana, teniendo como referencia los datos estadísticos de deserción escolar y la criminalidad proveniente de los adolescentes.

En el ámbito español, De la Rosa Cortina (s.f) publicó un artículo de revisión de tema, donde expone una serie de causas sociales que influyen en la problemática social de la delincuencia juvenil. Se habla así, de familias desestructuradas que no están en la capacidad de cumplir con el deber de formar a sus hijos respecto a normas, ética y cultura de valores. Las razones se hallan en que los padres o cuidadores poseen problemas de drogadicción, alcohol, pobreza, prostitución, enfermedades mentales. En otros casos, se trata de familias marginales o desarraigadas donde alguno de los dos padres no se encuentra presente.

Asimismo, el autor incurre en las repercusiones de la delincuencia juvenil, las tendencias de futuro en cuanto a las reacciones de los ciudadanos frente a jóvenes delincuentes, y finalmente esboza unos tratamientos que se le deben brindar al individuo infractor. Apoyándose en los principios sobre los que descansa la legislación de menores en España, De la Rosa Cortina (s.f) indica que el tratamiento para el menor delincuente requiere de datos familiares, sociales, psicológicos y educativos. Con ello se conformaría un equipo técnico especializado, donde se integre la labor de psicólogos, educadores y trabajadores sociales que asesoren a jueces y fiscales para efectuar el respectivo proceso.

Por otra parte, en el contexto local, Díaz, Pinilla, Vásquez, Asprilla \& Munive (2011), en su condición de integrantes de la Policía Nacional, realizaron una labor investigativa en la ciudad de Barranquilla, Colombia, sobre la incidencia que puede tener la pertenencia a una pandilla. El estudio indica que este podría ser el primer peldaño en la carrera delictiva de una persona. Eventualmente, los pandilleros pueden ser utilizados como mano de obra del crimen organizado. Las pandillas y las barras bravas son, además, expresión de las disfuncionalidades familiares, de la escuela y de la sociedad. Por todas esas razones, es importante analizar y comprender bien el fenómeno, para tener claridad sobre cómo abordarlo.

Otro estudio examinado fue el realizado por la Fundación Proceder Siglo XXI (2012), en alianza con la Universidad del Atlántico, Universidad del Norte y Policía Nacional. El trabajo estuvo encaminado hacia la caracterización de un grupo representativo de jóvenes que hacen parte de diferentes barras y pandillas de la ciudad o se encuentran en riesgo de ingresar a ellas. Se determinó además, la ubicación, identificación y caracterización de las principales pandillas que existen en el Distrito de Barranquilla.

Por último, respecto de los índices de delincuencia en Barranquilla, Acero Velásquez (2008) suministra algunos datos estadísticos en los que se plantea la situación. El reporte se sintetiza a continuación: En términos de tasas de homicidios por cada 100.000 habitantes del año 2007, Barranquilla es menos violenta que ciudades como Ibagué, Medellín, Armenia, Cali y Cúcuta, entre otras. Sin embargo, en relación con las ciudades capitales de los departamentos de la Costa, se sitúa por encima de San Andrés, Sincelejo, Cartagena, Montería, y por debajo de Riohacha, Valledupar y Santa Marta. En este sentido, si se compara a Barranquilla con los demás municipios del Área Metropolitana en términos de tasa de homicidios, se tiene que Galapa es el municipio que más ha logrado reducir este indicador, seguido de Soledad y Barranquilla.

Consecuentemente, en términos de la concentración de este delito en la ciudad, se tiene que en el 8.7\% de los barrios que componen la ciudad (según datos suministrados por la Administración, la ciudad tiene 207 barrios) ocurren el 50\% de los homicidios y en el 23\% ocurren el $80 \%$ de los casos, estando Rebolo con 8,05\%, Santo Domingo de Guzmán con 12,07\%, El Bosque con 15,80\%, El Centro $18,97 \%$ y Chiquinquirá $21,84 \%$, los barrios con mayor número de homicidios.

De igual forma, las lesiones personales han aumentado en los últimos tiempos. El 50\% de las lesiones se concentran en el $9.7 \%$ de los barrios que tiene Barranquilla, siendo Rebolo, El Centro, Ciudadela 20 de Julio y San Roque, La Chinita, El Bosque y Carrizal los más afectados, mientras el 50\% de los 
hurtos a personas se concentran en el 9,6\% de los barrios que tiene Barranquilla y el $80 \%$ de los casos se concentra en el $27 \%$ de los barrios, siendo Rebolo, el Centro y Pardo los barrios más afectados. En consecuencia de la revisión de antecedentes científicos, se llegó a la conclusión de que el delincuente juvenil es una persona con muchas carencias, necesidades y conflictos. Por tanto, si los riesgos no son controlados a tiempo, es factible la proliferación de jóvenes con un futuro incierto, menores de edad susceptibles de caer fácilmente en las drogas y por ende, candidatos a la transgresión con una existencia precaria y marginal. Esto, con el agravante de que las autoridades, entes gubernamentales $u$ otros estamentos a nivel mundial no hayan hasta la fecha, encontrado una solución efectiva al problema.

\section{METODOLOGÍA}

Para dar cumplimiento a las metas expuestas, el abordaje del problema se realizó desde un enfoque socio - crítico, acudiendo a un tipo de Investigación Acción Participación (IAP), con un diseño de campo que abarcó cuatro momentos o fases, a saber:

a) Fase descriptiva, que operativamente inició con los procesos de sensibilización y culminó con el levantamiento del perfil de los jóvenes infractores y los factores que los predisponen a delinquir. Así mismo, se determinó que resultados han dado las políticas, programas y estrategias implementados por las autoridades y organismos gubernamentales.

b) Fase propositiva, durante la cual se formularon acuerdos para un trabajo colaborativo intersectorial entre representantes de las unidades policiales responsables de la investigación (ESANA - MEBAR) y los líderes de las principales organizaciones y grupos afectados por la delincuencia juvenil, o con injerencia en la problemática.

c) Fase de intervención, que implicó la delimitación y conceptualización sobre las líneas de acción pertinentes a estrategias y planes para prevenir y combatir la delincuencia juvenil en Barranquilla y área metropolitana. Con algunas limitaciones, se efectuaron mesas de trabajo y actividades en las que participó un grupo de sujetos en proceso de reeducación.

d) Fase de evaluación, por la cual se analizaron los resultados derivados del trabajo colaborativo, se validaron las propuestas y se valoran los logros y debilidades. Posteriormente, se trazaron los ajustes correspondientes para que las líneas formuladas pudieran servir de base a una propuesta de intervención que apunte a resultados efectivos en Barranquilla y su área metropolitana, pero que también a futuro pueda ser extrapolada a otros escenarios en Colombia.

Para la recolección y procesamiento de información se acudió a las siguientes técnicas e Instrumentos: Encuesta diagnóstica, Revisión documental, Observación participante, Entrevista semiestructurada, reuniones y talleres participativos (Grupos focales) y específicamente, trabajo colaborativo.

La población de referencia que se tomó para la ejecución de la propuesta presentada fue de 1.179.000 habitantes de Barranquilla, según el último censo poblacional. Dado que se trataba de un gran número de unidades, se focalizó el trabajo en las zonas vulnerables de la ciudad (Mapa de riesgos de delincuencia). En esas comunidades se ubicó a los gestores de las alternativas de solución pertinentes a la magnitud del problema.

En relación con la muestra poblacional para la encuesta diagnóstica se tomaron 250 informantes residentes en las cinco (5) zonas de Barranquilla y en los cuatro (4) municipios del Área Metropolitana: Soledad, Malambo, Galapa y Puerto Colombia. Principalmente, se tuvieron en cuenta, tres características que sirvieron para estructurar el proceso, y que se mostrarán a continuación en los gráficos.

\section{Gráfico 1. Lugar de residencia}

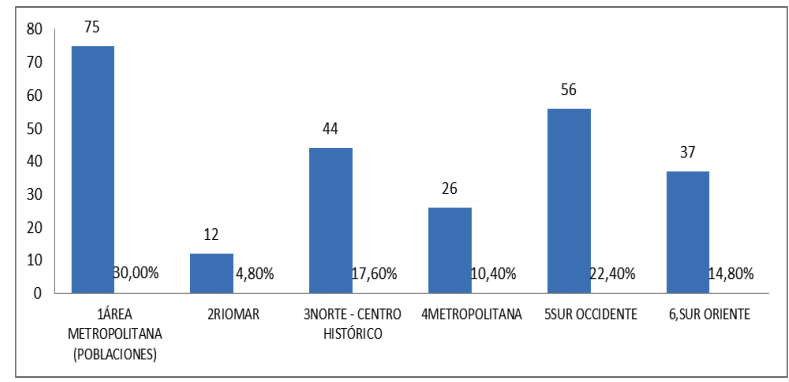


Gráfico 2. Rango de edades

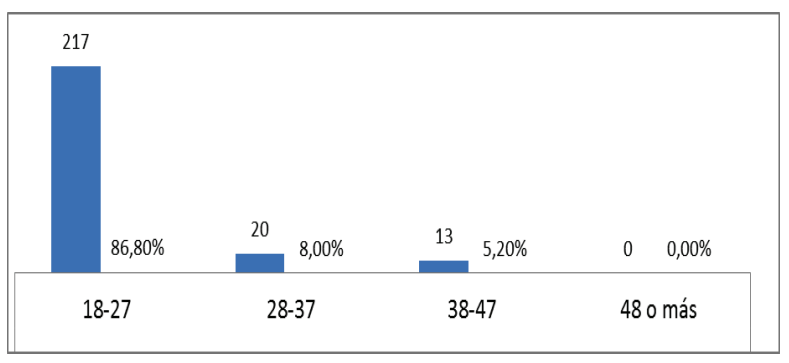

\section{Gráfico 3. Nivel educativo}

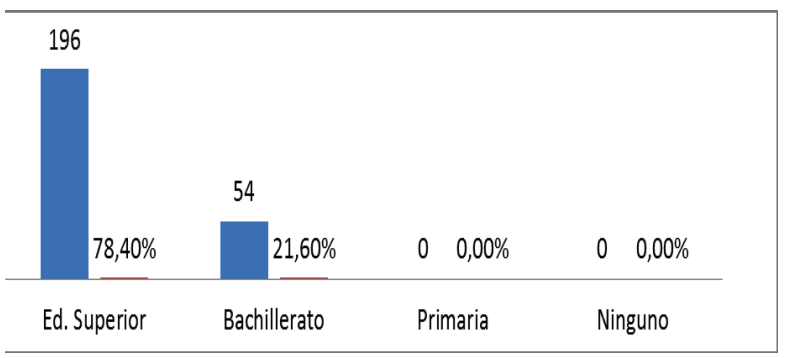

Fuente: encuesta realizada a 250 ciudadanos de la ciudad de Barranquilla y área Metropolitana

\section{Resultados}

3.1. Identificación del perfil de los jóvenes infractores y su asociación a factores de riesgo para conductas delictivas

En cumplimiento del primer objetivo específico trazado, se logró identificar el perfil de los jóvenes infractores y su asociación a factores de riesgo para conductas delictivas.

\section{Gráfico 4. Promedio de personas que viven una misma casa}

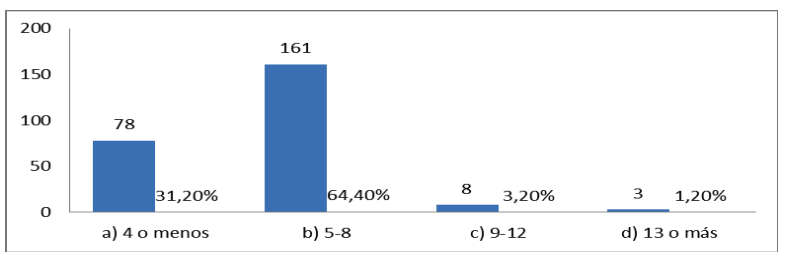

Fuente: encuesta realizada a 250 ciudadanos de la ciudad de Barranquilla y área Metropolitana

Como lo muestra la gráfica 4 , de acuerdo con la población encuestada, el promedio de residentes en un $64,40 \%$ de hogares es de 5 a 8 personas. Al analizar la procedencia de los informantes, se halló que en su mayoría, esta respuesta corresponde a los sectores del área metropolitana, suroccidente y suroriente de Barranquilla. Este dato da cuenta de la situación en la que gran parte de la ciudadanía está inmersa; es decir, el hacinamiento y la falta de recursos para subsistir. Se infiere que entre más miembros integren un mismo núcleo familiar, mayor será la disminución en su calidad de vida y el riesgo de los menores de edad a buscar formas ilícitas de abastecer sus necesidades.

\section{Gráfico 5. Empleo fijo o estabilidad laboral}

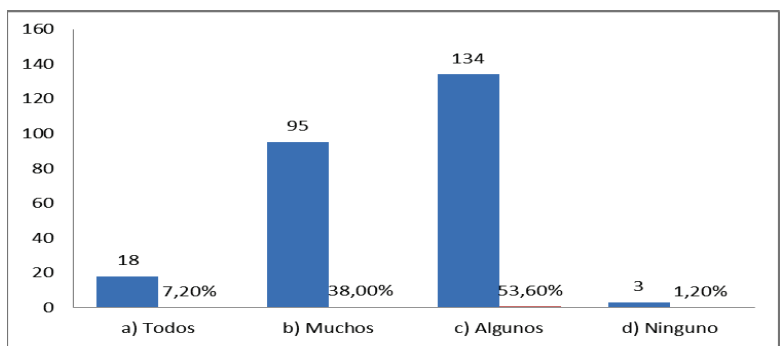

Fuente: encuesta realizada a 250 ciudadanos de la ciudad de Barranquilla y área Metropolitana

En lo que concierne a la estabilidad laboral, puede observarse que esta se encuentra entre $53,60 \%$ para algunos de los habitantes y 38,00\% para muchos de ellos. Según estos datos estadísticos, en la mayor parte de los hogares no todos los adultos se encuentran laborando. Este factor económico es de gran relevancia, teniendo en cuenta que muchas de estas familias pertenecen a estrato bajo y medio bajo. Como tal, se infiere dificultades para conseguir recurso financiero necesario para obtener alimentos, pagar recibos, comprar bienes necesarios para la supervivencia, cubrir servicios básicos, de salud y educación, entre otros, con lo cual se afecta la calidad de vida.

La falta de un trabajo estable y una vivienda propia y de espacios públicos óptimos, constituye en Colombia, uno de los problemas sociales más sensibles y complejos de resolver. Es imprescindible el diálogo, la concertación, el trabajo en equipo, aún más tomando en cuenta la nueva coyuntura política y la posibilidad de que la constitución pueda legitimar de manera precisa, no solamente en enunciado, el derecho a la vivienda de las familias colombianas. Amerita el asunto, compromisos por parte de distintos sectores de la sociedad, a fin de garantizar la igualdad de derechos de todos los ciudadanos, y sobre todo de la población menor de 18 años, que ante la falta de oportunidad se encuentra en riesgo permanente de delinquir. 
Gráfico 6. Comportamientos groseros por parte de los menores

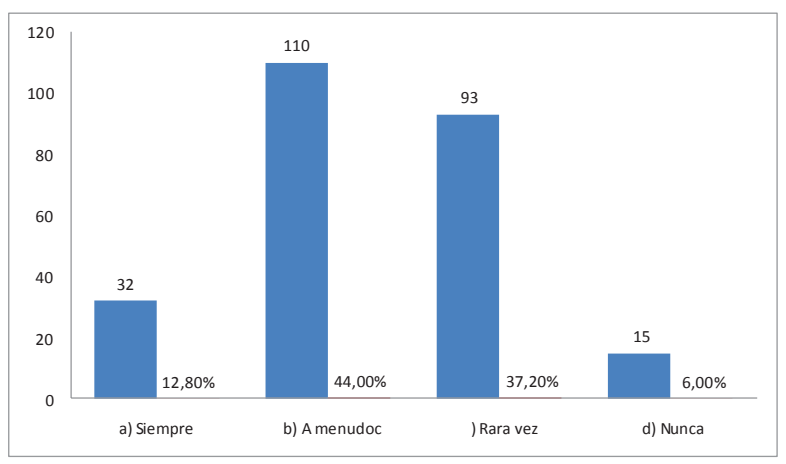

Fuente: encuesta realizada a 250 ciudadanos de la ciudad de Barranquilla y área Metropolitana

Si bien es cierto toda formación tiene su proceso, independientemente de las fluctuaciones que puedan existir, si en algún momento hay un choque emocional, esto puede generar lo que se conocen como vacíos o inestabilidades emocionales Esto sucede en los niños cuando sienten que sus padres no les prestan la atención. Es por ello, que muchas veces se tornan agresivos, insolentes e irrespetuosos, pero lo hacen generalmente para llamar la atención de sus progenitores o cuidadores, o para revelarse ante ellos. En Barranquilla y su área metropolitana el 44,00\% de los menores se comportan de forma grosera, lo cual resulta bastante abrumador, porque cada muestra de insolencia o irrespeto por parte de ellos presupone un decaimiento en los valores sociales y la predisposición a infringir las leyes.

\section{Gráfico 7. Actividades de aprovechamiento del tiempo libre}

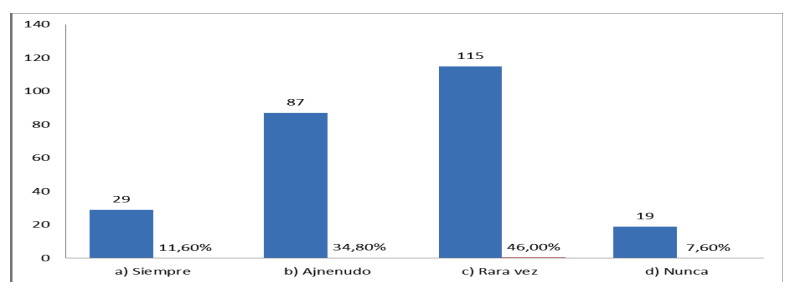

Fuente: encuesta realizada a 250 ciudadanos de la ciudad de Barranquilla y área Metropolitana

Según los datos estadísticos, el 46,00\% de los menores en Barranquilla y su área metropolitana, rara vez realizan actividades de aprovechamiento del tiempo libre (Cultura, recreación y deporte, y cerca de un $8 \%$ nunca lo hace. Estas cifras resultan preocupantes, dado que es una mayoría 53,6\% quienes no invierten su tiempo libre de forma adecuada, $y$ es sabido que esto puede llevarlos a cometer actos indebidos, e introducirse al mundo de las drogas. Amerita entonces, que se dé la asignación de espacios al aire libre en los que los menores puedan recrearse, aprovechar el tiempo de manera adecuada para evitar caer en el abismo de la delincuencia juvenil y consumo de sustancias psicoactivas.

Colombia es un país de contrastes, pues al tiempo de ser uno de los más inequitativos e inestables; ostenta sitiales privilegiados en cuanto a megadiversidad, multiculturalidad y creatividad. Esto último debería verse reflejado de manera holística en la calidad de vida de su población.

\section{Gráfico 8. Menores de edad fuera de sus vivien- das a altas horas de la noche}

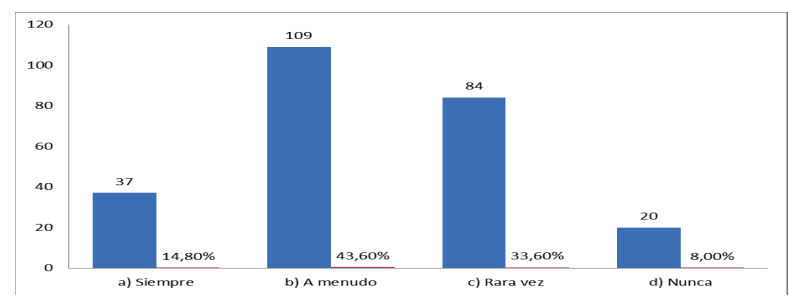

Fuente: encuesta realizada a 250 ciudadanos de la ciudad de Barranquilla y área Metropolitana

A nivel general, en la población encuestada, las respuestas apuntaron a que en Barranquilla y su área metropolitana, es usual que los niños y jóvenes se encuentren fuera de sus casas a altas horas de la noche. Un acumulado del 58,40\%, así lo corrobora. Estos casos generalmente se dan porque ellos viven en hogares disfuncionales, con padres separados o donde el afecto, atención y apoyo de estos no es perceptible. No hay control sobre los menores, y a menudo, estos no rinden cuentas de lo que hacen cuando están en la calle. De tal modo, la falta de autoridad, o en algunos casos, el libertinaje, hace que la situación se intensifique aceleradamente.

Gráfico 9. Menores de edad involucrados en actos delictivos o en riesgo de caer en grupos delincuenciales (bandas, pandillas, etc.)

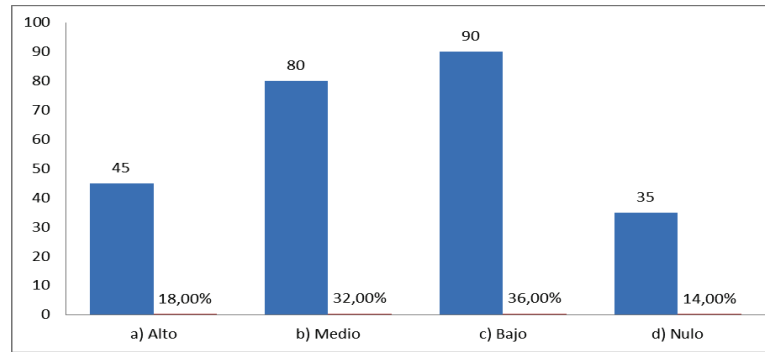

Fuente: encuesta realizada a 250 ciudadanos de la ciudad de Barranquilla y área Metropolitana 
Existe una relación altamente significativa entre la interacción del adolescente con amigos consumidores y su propio consumo. Se ha demostrado que la ingesta de drogas en los adolescentes, tiene como causa probable la compañía, o la influencia de amigos consumidores en oposición a los que no lo son. Entre los delincuentes juveniles, existen normas, y todo sujeto que pertenezca a ese grupo deberá adaptarse a ellas.

Las drogas pueden transformarse en un símbolo del grupo. Las relaciones amistosas implicarán unos determinados comportamientos que serán vistos como elecciones personales y no como una influencia social. Por tanto, la influencia de las "malas" compañías se hace más difícil de definir. Para Barranquilla y su área metropolitana, los datos estadísticos arrojan que los menores de edad y jóvenes, se encuentran entre un nivel alto $(18,00 \%)$ y medio $(32,00 \%)$ de caer en el abismo de la delincuencia y todo lo que esta conlleva, y aunque el nivel bajo tiene un $36,00 \%$ los otros dos niveles resultan relevantes, puesto que, equivalen al 50\% de la población que intenta

\section{Gráfico 10. Actos con los que los jóvenes del sec- tor empiezan una carrera delictiva}

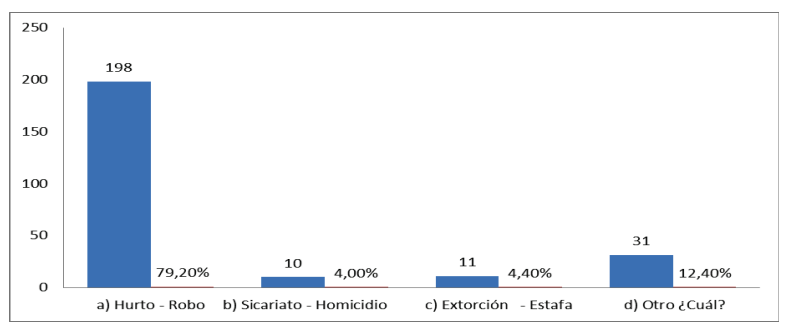

Fuente: encuesta realizada a 250 ciudadanos de la ciudad de Barranquilla y área Metropolitana

En lo que respecta a este dato estadístico; se puede apreciar, que Barranquilla y su área metropolitana, el 79, 20\% de los jóvenes empieza su carrera delictiva hurtando o robando. Del mismo modo, se establece que más del $50 \%$ de la población juvenil ha estado involucrado en actos delictivos de diferente índole, tales como, hurto, robo, sicariato, homicidio, extorsión, estafa. En ese sentido, cabe notar que muchos de los adolescentes y jóvenes que realizan esos actos ilícitos pertenecen a más de una modalidad de grupos violentos entre los que se pueden mencionar las pandillas barriales.

La incursión de los jóvenes, en la carrera delictiva se debe principalmente a la falta de políticas de prevención, pero sobre todo, al descuido de los padres, porque este es el punto de partida de la delincuencia. Es en el hogar mismo que se deben dar las bases principales para que se creen en los jóvenes el sentido de la responsabilidad, y afiancen sus valores para que el día de mañana sean personas de bien.

Queda claro que el problema de la delincuencia juvenil en Barranquilla y su área metropolitana, está asociado primordialmente, a factores de índole familiar, por ser esta unidad la base de la sociedad. A cada miembro del hogar compete una responsabilidad que implica actuar con base en sus costumbres como producto de elementos culturales, políticos y sociales. Asimismo, la educación, a través de las instituciones escolares, cumple un rol importante en la prevención del fenómeno, al estar obligados a fortalecer una formación integral en los niños, adolescentes y jóvenes que transitan por sus aulas. Finalmente, la existencia de políticas y programas del Estado, bajo el impulso de los entes gubernamentales, se constituye en una variable clave en cuanto al impulsar bienestar comunitario y calidad de vida a los ciudadanos, es más factible contrarrestar la aparición y proliferación de la delincuencia a temprana edad.

A manera de conclusión parcial; en relación con el primer objetivo específico de la investigación, quedó sentado que este flagelo afecta en gran medida, a la ciudadanía en general. En paralelo, se desglosaron los motivos que impulsan a los menores de edad y jóvenes a formar parte de grupos delincuenciales. Se auscultó si el problema tiene que ver con crisis de valores, inestabilidad familiar $\mathrm{u}$ otros factores directos o colaterales, en tanto preocupa que la falta de control a estos siga desencadenando funestas situaciones. Sin embargo, se detectó que el punto más crítico de la problemática se encuentra en la desescolarización de los niños y jóvenes, y en el escaso aprovechamiento del tiempo libre en actividades deportivas, recreativas, culturales y similares.

\subsection{Características, alcances y resultados de las políticas y acciones implementadas por las comu- nidades y estamentos públicos y privados para contrarrestar la delincuencia juvenil.}

Con miras al logro del objetivo específico número dos, el cual apuntó a reseñar las características, alcances y resultados de las políticas y acciones implementadas por las comunidades y estamentos públicos y privados para contrarrestar la delincuencia juvenil; los hallazgos arrojaron lo siguiente: 
Gráfico 11. Entidades que desarrollan programas de prevención en su comunidad

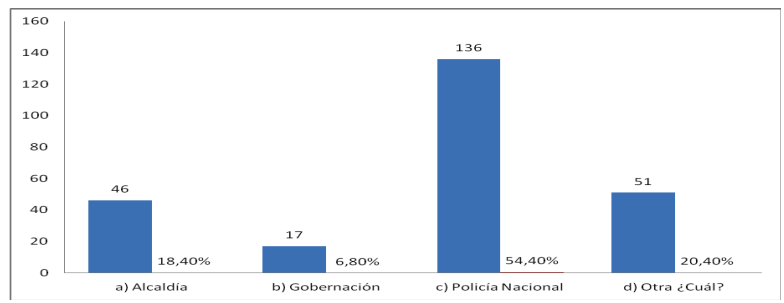

Fuente: encuesta realizada a 250 ciudadanos de la ciudad de Barranquilla y área Metropolitana

El 54,40 \% de la población de muestra, indica que los programas de prevención que se realizan en su comunidad son liderados por la Policía Nacional. Aquí cabe la pregunta: ¿Dónde están los demás entes gubernamentales y sociales? Como es bien sabido, la Policía Nacional, es un cuerpo armado, permanente, de naturaleza civil a cargo del Estado, encaminado a mantener y a garantizar el libre ejercicio de las libertades públicas y la convivencia pacífica de todos los habitantes del territorio nacional. Pero no le corresponde sólo a la Policía velar por el bienestar de la comunidad de Barranquilla y su área metropolitana. Los informantes insisten en que la Alcaldía, en alianza con la Gobernación del Atlántico, también debería liderar programas que estén encaminados hacia la protección del bienestar de todos y cada uno de los ciudadanos.

Gráfico 12. Los actuales planes y estrategias gubernamentales son efectivos frente a la delincuencia juvenil en Barranquilla

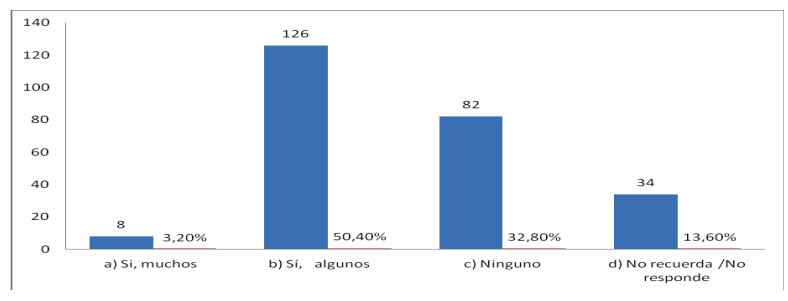

Fuente: encuesta realizada a 250 ciudadanos de la ciudad de Barranquilla y área Metropolitana

Como se observa, solo un $3,20 \%$ de la ciudadanía Barranquilla y su área metropolitana, reconoce la efectividad de los actuales planes y estrategias gubernamentales son efectivas frente a la delincuencia juvenil, mientras que un 50,40\% dice que solo algunos planes y estrategias funcionan. Frente al 32, $80 \%$ de la población que niega conocer algún plan o estrategia empleada por el gobierno, $\mathrm{o}$ que no los recuerda $(13,62 \%)$, la conclusión es que aunque si hay programas estatales para prevenir y contrarrestar el problema, estos no han logrado impactar en el imaginario de los ciudadanos.

Los informantes reclaman por soluciones urgentes y asertivas a un tema de seguridad pública. Sugieren a través de sus comentarios, una alianza estratégica entre la Alcaldía de Barranquilla, la Gobernación y la Policía Nacional, con el concurso de entidades educativas y estamentos sociales que puedan aportar al abordaje y solución del problema. Todo ellos, mediado por un trabajo fuerte intersectorial e interdisciplinario que tome en cuenta la participación de la comunidad afectada, de jóvenes en riesgo y de infractores con voluntad de resocialización.

En síntesis, en virtud del segundo objetivo específico se determinó que los problemas de delincuencia que se dan en la población auscultada, están ligados al desconocimiento que tienen los ciudadanos sobre la existencia de programas ejecutados por las autoridades, entes públicos y privados y organismos sociales. No obstante, gran parte de la muestra identifica los programas comunitarios liderados por la Policía Nacional, en materia de prevención y control de la delincuencia juvenil. Sin embargo, insisten en que son los órganos de Gobierno quienes tienen la obligación de velar por el bienestar de la comunidad. Señalan concretamente, que la Alcaldía, en alianza con la Gobernación, debería liderar programas encaminados a la protección del bienestar de todos y cada uno de los ciudadanos.

3.3. Líneas de acción para el diseño e implementación de programas, planes y estrategias de atención, prevención y control de la delincuencia juvenil en contextos de alta vulnerabilidad.

En el presente ítem se refleja el alcance del objetivo específico número tres, que consistió en delimitar mediante un trabajo colaborativo -intersectorial y comunitario- liderado por la policía nacional, las líneas de acción para el diseño e implementación de programas, planes y estrategias de atención, prevención y control de la delincuencia juvenil en contextos de alta vulnerabilidad.

El trabajo de prevención amerita una alianza estratégica que incluya a todos los sectores de la sociedad, incluyendo a la población afectada pues todos hacen parte del problema y como tal, tienen mucho por decir y decidir. En torno a las líneas de acción que se requiere abordar, el punto de partida es el fortalecimiento del núcleo familiar respecto del componente socio afectivo y una sólida formación en valores. 
En segunda instancia, es preciso que se haga incursión directa en la comunidad, desarrollando actividades de sensibilización, recreación y capacitación a los niños y jóvenes; esto es, poner en marcha programas mediante los cuales, ellos se sientan importantes y puedan canalizar todas sus energías de manera sana, contrarrestando por iniciativa propia, los riesgos de delincuencia latentes a su alrededor. Por último, y no lo menos importante, resulta clave la labor de las escuelas porque es allí donde los niños y jóvenes tienen la mayor oportunidad de sociabilización y por tanto, tienden a asimilar conductas y moldear su personalidad con base en su afinidad con personas - compañeros o amigos - con quienes comparten gran parte de su tiempo o le inspiran admiración por cualquier razón.

\subsubsection{Familia}

Se debe trabajar con las familias de los jóvenes a través de:

- Sensibilización y concientización a los padres, madres u familiares a cargo de los niños y jóvenes mediante charlas y reuniones colectivas.

- Crear espacios de reflexión y debate, con la idea de que sea un proceso en el que la acción y la reflexión sean paralelos y estén dentro de un mismo eje común.

- Seminarios de fortalecimiento en valores

- Foros sobre ¿Cómo ayudar a los jóvenes a no caer en el abismo de la delincuencia?

\subsubsection{Comunidad}

Con esta se pretende trabajar en:

- Actividades de sensibilización, recreación y capacitación a los niños y jóvenes.

- Formación de Escuela de futbol

- Puesta en marcha una microempresa de manualidades en yeso, producto de las capacitaciones en emprendimiento e ideas de negocios.

- Lanzamiento del proyecto Actuar 20 "Creación de una microempresa con jóvenes en condición de alta vulnerabilidad". Fabricación de escobas y traperos en barrios Primero de Mayo y Ferias en Soledad, Atlántico.

\subsubsection{Escuela}

La escuela es el espacio en el cual, los individuos se desarrollan personal y socialmente; la capacidad de ser una persona socialmente responsable tiene origen primeramente en la formación que da la familia como ya se ha mencionado, sin embargo, las escuelas tienen la capacidad de enfocar su enseñanza a través de actividades formativas que primeramente hagan al alumno interiorizar el valor de él mismo y el respeto para con ello generar que de manera autónoma haga lo propio por la sociedad y el medio que lo rodea.

Las acciones que se adoptan en la solución de problemas de los niños y jóvenes deben alentar a los gobiernos y a las instancias que las emprenden para que sean más sensibles a las aspiraciones que tienen estos jóvenes para un mundo mejor y para que tomen en cuenta las demandas encaminadas a formar parte de la solución en vez de formar parte del problema. Para ello, se requiere: a) Promover una formación más amplia y específica en cuestión de valores; b) Hacer un trabajo informativo, para que las escuelas sensibilicen a los jóvenes mediante charlas, debates, conversatorios, etc. ) Brindar apoyo psicológico para reorientar a los niños y jóvenes.

Se colige entonces; que en cumplimiento del tercer objetivo específico se emprendió un trabajo colaborativo -intersectorial y comunitario- liderado por la Policía Nacional. El punto clave fue trazar las líneas de acción para el diseño e implementación de programas, planes y estrategias de atención, prevención y control de la delincuencia juvenil en contextos de alta vulnerabilidad. Se definió entonces, que son tres los focos de atención para el abordaje e intervención del problema. En primer lugar, la familia, en tanto se requiere el fortalecimiento del núcleo en términos del componente socio afectivo y formación en valores. En segunda instancia, la comunidad, pues se requiere desarrollar actividades de sensibilización, recreación y capacitación a niños, adolescentes y jóvenes infractores y en riesgo. Por último, y no lo menos importante, resulta clave la labor de la escuela, porque es allí donde los menores de edad tienen la mayor oportunidad de sociabilización y aprendizaje integral.

\section{Conclusiones}

Mediante la investigación reseñada, se pudo corroborar que en los distintos sectores de Barranquilla, pero sobre todo en los barrios de estrato socioeconómico bajo y municipios del área metropolitana; un gran porcentaje de la población con edades entre los 12 y los 24 años - especialmente menores de edad - se halla en situación de riesgo frente al fenómeno de la delincuencia juvenil. Esto se debe principalmente, a un núcleo familiar disfuncional, 
o desintegrado por la falta de una vivencia plena de valores y principios, de orden moral y ético respectivamente. A la par, las dificultades de muchos hogares en materia de servicios adicionales a los básicos y la tendencia al hacinamiento y a una economía informal enraizada en la carencia de estabilidad laboral por parte de los adultos, genera un ambiente poco favorable para el desarrollo integral del ser.

No obstante, el punto más crítico de la problemática se encuentra en la desescolarización de los niños y jóvenes, y en el escaso aprovechamiento del tiempo libre en actividades deportivas, recreativas, culturales y similares, bien sea por la falta de espacios o por el desconocimiento de programas sociales liderados por los entes gubernamentales, la fuerza pública u organizaciones civiles con injerencia en el tema. Como consecuencia, en esta zona del país es usual la presencia de niños y jóvenes en las calles a altas horas de la noche, hecho que sumado a otras condiciones materiales, psicosocioafectivas y legales los hace vulnerables tanto al consumo de alcohol y alucinógenos, como al ingreso a pandillas y grupos delincuenciales.

Los alcances y resultados de las políticas, leyes, estrategias y planes adoptados por las autoridades locales y departamentales y otras instancias de índole educativo, social y jurídico, no logran ser visibles para la ciudadanía. Por tal razón, para que las medidas tengan la efectividad, reconocimiento e impacto que se espera; es preciso trabajar de manera intersectorial e interdisciplinaria en su diseño e implementación y que atiendan, más que a la represión y al castigo de los jóvenes infractores, a la prevención de la delincuencia desde edades tempranas.

Como resultado visible del proyecto, se dio un diagnóstico de la delincuencia juvenil en Barranquilla y su área Metropolitana, y consecuentemente, una descripción de las necesidades que se detectan en materia de atención, prevención y control de este flagelo. En ese orden de ideas, el presente informe de investigación esboza el fenómeno de forma global, pero apuntando concretamente a algunos aspectos que lo tipifican. Se alude así, a la carencia de afecto por parte de la familia, a dificultades socioeconómicas, a la influencia de los amigos, y por supuesto, a la inmersión de los jóvenes en las pandillas y las drogas, así mismo, las implicaciones que dicha problemática tiene en la sociedad.

Por consiguiente, se podría decir, que la educación, los valores éticos y morales que se le inculquen a una persona, serían primordiales para lograr su plena madurez como individuo en interacción con una colectividad. Quiere decir esto, que toda formación tiene un proceso que permite que el ser entre en una etapa de plenitud, y a su vez de confrontaciones. Si dicho proceso se trunca en algún momento, se podrían crear vacíos e inestabilidades en el desarrollo armónico e integral del sujeto a un grupo social.

En la consecución de esta meta, la policía nacional juega un rol fundamental, dado que su tarea no se limitaría a salvaguardar la seguridad ciudadana, ejercer la autoridad y ejecutar los procedimientos necesarios para judicializar al delincuente. Su misión estaría focalizada en intervenir en procesos participativos de reeducación y resocialización de los infractores de la ley, y asimismo, a partir de la identificación de las zonas vulnerables y los factores asociados a la delincuencia juvenil, hacer un acompañamiento a los jóvenes en situación de riesgo. Ello se logra con el conocimiento de la comunidad, acercándose más a esta, no mostrándosele al ciudadano autocráticamente, sino dándole confianza para el establecimiento de unas buenas relaciones interpersonales. 


\section{Referencias}

Acero Velásquez (2008). Violencia y Delincuencia: Barranquilla, diagnóstico y recomendaciones. Alcaldía de Barranquilla: disponible en http:/ / www.barranquilla.gov.co/documentos/Informe_web_2.pdf

De la Rosa Cortina, J. (s.f). El fenómeno de la delincuencia juvenil: causas y tratamientos. P. 1- 14. Recuperado el 27 de septiembre de 2013 disponible en http:/ / www.encuentros-multidisciplinares.org/ Revistan\%BA13/Jos\%E9\%20Miguel\%20de\%20la\%20Rosa\%20Cortina.pdf

Díaz, et al (2011). Innovación en el servicio policial una alternativa para la intervención en casos de pandillas en los Barrios marginales de Barranquilla. Escuela Antonio Nariño, Barranquilla, Colombia.

Fundación Proceder Siglo XXI (2012). Intervención psicosocial a jóvenes en situación de riesgo pertenecientes a pandillas en el Distrito de Barranquilla. Recuperado el día 15 de Octubre de 2103 disponible en https://www.google.com.co/url?sa=t\&rct=j\&q=\&esrc=s\&source=web\&cd=2\& $\mathrm{cad}=$ rja\&sqi=2\&ved $=0 \mathrm{CDQQFjAB} \& u r l=\mathrm{http} \% 3 \mathrm{~A} \% 2 \mathrm{~F} \% 2 \mathrm{Fwww} \cdot$ barranquilla.gov.co $\% 2 \mathrm{Findex}$. php\%3Foption\%3Dcom_docman\%26task\%3Ddoc_download\%26gid\%3D1885\%26Itemid\%3D126 \&ei=Ka0_Uor9N8Wn4APVt4DgBQ\&usg=AFQjCNHJv9Bml4glMoiOveHh0iDd9PbrA\&sig2=MIy qH5E_Hj-AAd-nzb_1Jw

Garrido, G. V. (1987). Delincuencia juvenil. Madrid: Alhambra

Monroy, J. A. (1998). Obras completas, tomo III. Terrassa, España: CLIE

Páez Morales, O. (2013). Delincuencia juvenil como fenómeno que afecta la convivencia y seguridad ciudadana. Bogotá D.C., II ciclo. Escuela de postgrados de policía Miguel Antonio Lleras Pizarro, trabajos de interés institucional.

Presidencia de la República de Colombia. (Marzo 2007). ABC del Código de Infancia y Adolescencia. Recuperado 27 de septiembre de 2013, de ABC del Código de Infancia y Adolescencia: disponible en https: / / docs.google.com/viewer? $\mathrm{a}=\mathrm{v} \& \mathrm{q}=$ cache:JMXLk6z9XOkJ:wsp.presidencia.gov.co/Ninos / Documents / codigo.pdf + sistema + de+responsabilidad+penal + para+adolescentes\&hl=es\&pid=bl\& srcid=ADGEEShKfyavEdZg7pCnaa0rLTwfjcw1ludVNUKliNzowTDIj1MVItVHqBtpKhwbsGh0G 7oMSnKiYwLY5O_WfcfU1Hi8mW0D9bwbaB-O_6BSwrfolIR4z1p4tKURYFZAYYWIQgG_Hyx1\& sig=AHIEtbSpPvyZzyDFoVvSOYZ_8zDioW0XUw 